PROCEEDINGS OF THE

AMERICAN MATHEMATICAL SOCIETY

Volume 138, Number 8, August 2010, Pages 2873-2887

S 0002-9939(10)10369-4

Article electronically published on April 8, 2010

\title{
A GEOMETRIC INTERPRETATION OF THE CHARACTERISTIC POLYNOMIAL OF REFLECTION ARRANGEMENTS
}

\author{
MATHIAS DRTON AND CAROLINE J. KLIVANS
}

(Communicated by Jim Haglund)

\begin{abstract}
We consider projections of points onto fundamental chambers of finite real reflection groups. Our main result shows that for groups of types $A_{n}, B_{n}$, and $D_{n}$, the coefficients of the characteristic polynomial of the reflection arrangement are proportional to the spherical volumes of the sets of points that are projected onto faces of a given dimension. We also provide strong evidence that the same connection holds for the exceptional, and thus all, reflection groups. These results naturally extend those of De Concini and Procesi, Stembridge, and Denham, which establish the relationship for 0 -dimensional projections. This work is also of interest to the field of orderrestricted statistical inference, where projections of random points play an important role.
\end{abstract}

\section{INTRODUCTION}

A classic problem in statistics is the testing of hypotheses that impose orderrestrictions on a parameter vector or, more generally, require a parameter vector to belong to a polyhedral cone $\mathcal{C} \subseteq \mathbb{R}^{n}$; see for instance [RWD88, SS05]. Let $\pi_{\mathcal{C}}(x)$ be the orthogonal projection of $x \in \mathbb{R}^{n}$ onto $\mathcal{C}$. If the projection $\pi_{\mathcal{C}}(x)$ is in the relative interior of a $k$-dimensional face of $\mathcal{C}$, then we say that $\pi_{\mathcal{C}}(x)$ is $k$-dimensional. The following problem arises when studying the probability distributions of statistics suitable for testing membership in the cone $\mathcal{C}$ :

Problem 1. Which fraction of the unit sphere in $\mathbb{R}^{n}$, as measured by surface volume, is occupied by the points $x$ for which the projection $\pi_{\mathcal{C}}(x)$ is $k$-dimensional?

In statistical calculations, the surface volume fractions appear as weights in mixtures of probability distributions, most commonly, mixtures of so-called chisquare distributions. We denote the surface volume fractions by $\nu_{k}$ and refer to them as projection volumes.

Example 2. Let $\mathcal{C}=[0, \infty)^{2}$ be the non-negative orthant in $\mathbb{R}^{2}$. All points $x$ in the positive orthant $(0, \infty)^{2}$ lie inside the cone and thus have a 2 -dimensional projection

Received by the editors June 11, 2009.

2010 Mathematics Subject Classification. Primary 51F15, 05E15, 20F55, 62H15.

Key words and phrases. Characteristic polynomial, Coxeter group, hyperplane arrangement, order-restricted statistical inference, reflection group.

The first author was partially supported by NSF grant DMS-0746265 and an Alfred P. Sloan Research Fellowship. 
$\pi_{\mathcal{C}}(x)$. All points in the non-positive orthant $(-\infty, 0]^{2}$, the polar cone, are projected to the origin; that is, they have 0 -dimensional projection. All remaining points being 1-dimensional, the projection volumes are $\nu_{0}=\nu_{2}=1 / 4$ and $\nu_{1}=1 / 2$.

In small dimensions, Problem 1 is resolved easily. If $n=2$, as in Example 2, then the projection volume $\nu_{2}$ is determined by the angle between the two rays that span the cone, assuming the cone is pointed. The fraction $\nu_{1}$ is always equal to $1 / 2$, and $\nu_{2}=1-\nu_{0}-\nu_{1}$. If $n=3$, then finding the projection volumes turns into a problem of spherical trigonometry. For higher dimensions, however, the problem becomes more difficult, and statistical practice typically relies on Monte Carlo integration for calculating the projection volumes. However, one important exception is known.

Theorem 3. If $\mathcal{C}=\left\{x \in \mathbb{R}^{n}: x_{1} \leq x_{2} \leq \cdots \leq x_{n}\right\}$, then the projection volumes are proportional to the absolute values of the Stirling numbers of the first kind, that $i s$, the coefficients of the polynomial $\chi(t)=t(t-1)(t-2) \cdots(t-n+1)$.

In this paper we reprove and generalize this result to two other infinite families of polyhedral cones. The generalization is based on the fact that the cone $\mathcal{C}$ of Theorem 3 can be seen as a fundamental chamber of the reflection arrangement corresponding to the reflection group $A_{n-1}$.

Reflection (or Coxeter) groups are classical objects (see for example Hum90, ST54) and have recently received considerable attention from a more combinatorial perspective; see for example [BB05, BGW03, FR07. A fundamental chamber of a reflection arrangement in $\mathbb{R}^{n}$ is the polyhedral cone obtained by taking the closure of any one of the arrangement's regions. To any hyperplane arrangement, one may associate a polynomial defined over its intersection lattice, known as the characteristic polynomial of the arrangement. These notions will be introduced thoroughly in Section 3. Our main results, Theorems 18 and 19 (combined here), solve Problem 1 for certain families of reflection arrangements by relating projection volumes to coefficients of characteristic polynomials.

Theorem 4. Let $\mathcal{W}$ be a reflection group of type $A_{n}, B_{n}$, or $D_{n}$ and let $\chi(t)$ be the characteristic polynomial of the associated reflection arrangement. For a generic point $x$ in the fundamental chamber $\mathcal{C}$ of $\mathcal{W}$, the number of group elements $g \in \mathcal{W}$ with $k$-dimensional projection $\pi_{\mathcal{C}}(g x)$ is equal to the absolute value of the coefficient of $t^{k}$ in $\chi(t)$.

This yields the following corollary; see Lemma 9 and Remark 10 of Section 3

Corollary 5. Let $\mathcal{W}$ be a reflection group of type $A_{n}, B_{n}$, or $D_{n}$ and let $\chi(t)$ be the characteristic polynomial of the associated reflection arrangement. Then the projection volumes for any fundamental chamber of $\mathcal{W}$ are proportional to the absolute values of the coefficients of $\chi(t)$.

We conjecture that Theorem 4, and thus also Corollary [5, holds for any finite reflection group 1

Conjecture 6. Let $\mathcal{W}$ be a finite reflection group and let $\chi(t)$ be the characteristic polynomial of the associated reflection arrangement. For a generic point $x$ in the fundamental chamber $\mathcal{C}$ of $\mathcal{W}$, the number of group elements $g \in \mathcal{W}$ with

\footnotetext{
${ }^{1}$ Corollary 5 is now proved uniformly for all reflection groups. The proof considers more general arrangements and does not settle Conjecture 6 The result will appear in a forthcoming paper.
} 
$k$-dimensional projection $\pi_{\mathcal{C}}(g x)$ is equal to the absolute value of the coefficient of $t^{k}$ in $\chi(t)$.

As seen in Section 3 it is sufficient to prove Conjecture 6 for the irreducible reflection groups. By Proposition 13, Conjecture 6 is true for all reflection groups of rank 2. Theorems 18 and 19 prove Conjecture 6 for the three infinite families among the irreducible reflection groups of rank at least 3. We also offer strong empirical evidence for all but one of the remaining exceptional irreducible reflection groups. The one group not covered by our computer experiments is known as $E_{8}$, and it is not covered simply due to its large size. We strongly believe that Conjecture 6 also holds for $E_{8}$ and thus for all finite reflection groups.

Recent work by De Concini and Procesi, and Stembridge [DCP06] and subsequent work by Denham Den08 connects the projection volume for 0-dimensional projections to the bottom coefficient of the characteristic polynomial. Our Conjecture 6 provides a natural extension.

As we also point out in Remark 22, the geometric interpretation of the characteristic polynomial in terms of projection volumes is different from other interpretations that have appeared in the literature. It would thus be interesting to establish bijections between the different partitions of $\mathcal{W}$ that arise in the different interpretations.

\section{Statistical motivation}

Problem 1 is motivated by the problem of computing $p$-values in statistical hypothesis testing. We review the basics here for the interested reader; a detailed treatment can be found in monographs such as [RWD88, SS05].

Consider $n$ measurements $x_{1}, \ldots, x_{n}$ that are modelled as realizations of independent normal (Gaussian) random variables $X_{1}, \ldots, X_{n}$ with unknown means $\mu_{1}, \ldots, \mu_{n}$. Assume that the variances of the random variables are known and equal to a common value $\sigma_{0}^{2}$. Without loss of generality, we may assume $\sigma_{0}^{2}=1$. Given the observed point $x=\left(x_{1}, \ldots, x_{n}\right) \in \mathbb{R}^{n}$, we wish to test the hypothesis that the mean vector $\mu=\left(\mu_{1}, \ldots, \mu_{n}\right)$ belongs to some polyhedral cone $\mathcal{C}$.

For a concrete example, suppose we are measuring a patient's response to $n$ treatments that consist of taking a drug in increasing dosage. In this case, the cone from Theorem 3, representing the hypothesis that the unknown mean vector satisfies $\mu_{1} \leq \mu_{2} \leq \cdots \leq \mu_{n}$, is of interest.

Testing a hypothesis requires quantifying the evidence that the observed point $x$ provides against the considered hypothesis. To this end, one picks a measure of distance between $x$ and the cone $\mathcal{C}$. In the present context, a canonical choice is the squared Euclidean distance between $x$ and $\mathcal{C}$, which we denote by $d(x)$. If $d(x)$ is 'too large', then the hypothesis that the mean vector belongs to $\mathcal{C}$ is rejected.

In order to decide which distances ought to be considered large, a probability calculation is used. Let $d(X)$ be the squared Euclidean distance between the random point $X=\left(X_{1}, \ldots, X_{n}\right)$ and $\mathcal{C}$. Consider the maximum probability of the event that $d(X) \geq d(x)$ when $\mu \in \mathcal{C}$, that is, the probability

$$
p=\max _{\mu \in \mathcal{C}} P_{\mu}(d(X) \geq d(x)),
$$

which is also known as a $p$-value. We then declare the distance $d(x)$ to be 'too large' if $p$ is smaller than some chosen threshold. 
It is in computing the $p$-value that the projection volumes become relevant. Because of our assumption that the random variables are normally distributed, the $p$-value can be shown to be equal to the linear combination

$$
p=\sum_{k=0}^{n} \nu_{n-k} \cdot P\left(\chi_{k}^{2} \geq d(x)\right),
$$

where the weights are precisely the projection volumes. The symbol $\chi_{k}^{2}$ denotes a random variable that is distributed according to a chi-square distribution with $k$ degrees of freedom; that is, it is distributed like the sum of the squares of $k$ independent normal random variables with mean zero and variance one. Chi-square probabilities are readily available in tables or software, and the difficulty lies in determining the projection volumes.

\section{ReFleCtion ARRANGEMENTS}

In this section, we define the basic notions from the theory of hyperplane arrangements and finite reflection groups. For excellent references on these topics, we refer the reader to [Sta07] and Kan01.

3.1. Hyperplane arrangements. A (real central) hyperplane arrangement $\mathcal{A}$ is a collection of codimension-one linear subspaces of $\mathbb{R}^{n}$. All arrangements appearing in this paper are assumed finite. The rank of an arrangement $\mathcal{A}$ is defined to be the dimension of the linear space spanned by the normal vectors to its hyperplanes. Namely, if $\mathcal{A}=\left\{H_{1}, \ldots, H_{m}\right\}$ and $H_{i}=\left\{x \in \mathbb{R}^{n}: \alpha_{i} x=0\right\}$, where $\alpha_{i}$ is a non-zero vector in $\mathbb{R}^{n}$, then

$$
\operatorname{Rk}(\mathcal{A})=\operatorname{dim}\left(\operatorname{Span}\left\{\alpha_{1}, \ldots, \alpha_{m}\right\}\right) .
$$

An arrangement in $\mathbb{R}^{n}$ is said to be essential if the rank of $\mathcal{A}$ is $n$. A region or chamber of $\mathcal{A}$ is any connected component of the complement of the union of all the hyperplanes in $\mathcal{A}$. The closure of a chamber of any essential arrangement forms a pointed polyhedral cone. We will consider projections onto cones formed by particular classes of such hyperplane arrangements.

Much of the combinatorics of a hyperplane arrangement is encoded by its intersection lattice. Given an arrangement $\mathcal{A}$, let $L(\mathcal{A})$ be the set of all intersections of collections of hyperplanes in $\mathcal{A}$. We include $\mathbb{R}^{n}$ in $L(\mathcal{A})$ as the intersection of the empty collection. Define a partial order on $L(\mathcal{A})$ by reverse inclusion of intersections; that is, $x \leq y$ in $L(A)$ if $y \subseteq x$. Then $L(A)$ forms a lattice ranked by codimension $n-\operatorname{dim}(x)$. The Möbius function $\mu: L(\mathcal{A}) \rightarrow \mathbb{Z}$ of this lattice is defined recursively by $\mu\left(\mathbb{R}^{n}\right)=1$ and $\sum_{z \leq y} \mu(z)=0$. The characteristic polynomial of the hyperplane arrangement is the polynomial

$$
\chi_{A}(t)=\sum_{x \in L(\mathcal{A})} \mu(x) t^{\operatorname{dim}(x)} .
$$

We remark that the Poincaré polynomial $\pi(\mathcal{A}, t)$, as defined for example in [T92], is related to the characteristic polynomial by

$$
\chi_{\mathcal{A}}(t)=t^{\operatorname{Rk}(\mathcal{A})} \pi\left(\mathcal{A},-t^{-1}\right) .
$$

Example 7. Consider the hyperplane arrangement $\mathcal{A} \subset \mathbb{R}^{2}$ given by the two coordinate axes $H_{1}$ and $H_{2}$. The intersection lattice of this arrangement is $L(\mathcal{A})=$ $\left\{\mathbb{R}^{2}, H_{1}, H_{2},\{0\}\right\}$, with its elements ordered as $\mathbb{R}^{2} \leq H_{1} \leq\{0\}$ and $\mathbb{R}^{2} \leq H_{2} \leq\{0\}$. 
The Möbius function thus assigns the values $\mu\left(\mathbb{R}^{2}\right)=1, \mu\left(H_{1}\right)=\mu\left(H_{2}\right)=-1$ and $\mu(\{0\})=1$. The characteristic polynomial equals $\chi_{\mathcal{A}}(t)=t^{2}-2 t+1$.

3.2. Reflection groups. Let $\mathcal{W} \subset G L\left(\mathbb{R}^{n}\right)$ be a (finite) reflection group. Recall that a reflection in $\mathbb{R}^{n}$ is an isometry which fixes the points of some hyperplane $H$, often called the mirror of the reflection. A finite reflection arrangement or Coxeter arrangement is the collection of all mirrors of a finite reflection group. A fundamental chamber of the group $\mathcal{W}$ is the closure $\mathcal{C}$ of one of the regions in the reflection arrangement. Our main results, Theorems 18 and 19 below, establish a link between the coefficients of the characteristic polynomial of a reflection arrangement and the projection volumes of its fundamental chambers.

Example 8. The two coordinate axes in the plane discussed in Example 7 form a simple example of a reflection arrangement. The non-negative cone discussed in Example 2 is a fundamental chamber for this arrangement. The projection volumes $(1 / 4,1 / 2,1 / 4)$ for this chamber are proportional to the absolute values of the coefficients of the characteristic polynomial $t^{2}-2 t+1$.

The following lemma will provide us with a combinatorial approach to solving Problem 1 for fundamental chambers.

Lemma 9. Let $\mathcal{C}$ be a fundamental chamber of a finite reflection group $\mathcal{W}$. For $x \in \mathbb{R}^{n}$, let $b_{k}(x)$ be the number of group elements $g \in \mathcal{W}$ for which $\pi_{\mathcal{C}}(g x)$ is $k$-dimensional. If $b_{k}(x) \equiv b_{k}$ is constant outside a Lebesgue null set of choices for $x$, then $\pi_{\mathcal{C}}(X)$ is $k$-dimensional with probability $b_{k} /|\mathcal{W}|$ for any random vector $X$ whose joint distribution is continuous and invariant under the action of $\mathcal{W}$.

Proof. By the invariance of the distribution of $X$,

$$
P\left(\pi_{\mathcal{C}}(X) \text { is } k \text {-dim. }\right)=\frac{1}{|\mathcal{W}|} \sum_{g \in \mathcal{W}} P\left(\pi_{\mathcal{C}}(g X) \text { is } k \text {-dim. }\right)
$$

When summing up conditional probabilities, we obtain that

$$
\sum_{g \in \mathcal{W}} P\left(\pi_{\mathcal{C}}(g X) \text { is } k \text {-dim. } \mid X=x\right)=b_{k}
$$

for almost all $x \in \mathbb{R}^{n}$. Therefore,

$$
P\left(\pi_{\mathcal{C}}(X) \text { is } k \text {-dim. }\right)=\frac{1}{|\mathcal{W}|} \sum_{g \in \mathcal{W}} \int P\left(\pi_{\mathcal{C}}(g X) \text { is } k \text {-dim. } \mid X=x\right) d P^{X}(x)
$$

is equal to $b_{k} /|\mathcal{W}|$, as claimed.

Remark 10. A canonical choice for the random vector $X=\left(X_{1}, \ldots, X_{n}\right)$ in Lemma 9 is to pick $X_{1}, \ldots, X_{n}$ as independent standard normal random variables. Then the joint distribution of $X$ is invariant under the action of the orthogonal group and thus in particular invariant under the action of all reflection groups in $\mathbb{R}^{n}$. Moreover, for this choice, the probability that $X$ is $k$-dimensional is equal to the projection volume $\nu_{k}$ that appears in Problem 1 . 
3.3. Irreducible reflection groups. Finite reflections groups coincide with the finite Coxeter groups and as such there is a classification of the irreducible finite reflection groups; see for example [GB85, Hum90, Kan01. This classification contains four infinite families, typically denoted as $A_{n}, B_{n}=C_{n}, D_{n}$ and $I_{2}(m)$. As remarked earlier, there has been considerable attention recently on the combinatorics of Coxeter groups. A natural combinatorial perspective of these groups is in terms of symmetries; such a perspective will be important in our proofs of the main results. The group $A_{n}$ is the symmetry group of the $n$-simplex and is thus isomorphic to the symmetric group on $n+1$ characters. It acts by permutation of the entries of vectors in $\mathbb{R}^{n+1}$. The group $B_{n}$ is the symmetry group of the $n$-hypercube and consists of signed permutations of vectors in $\mathbb{R}^{n}$. The group $D_{n}$ is a subgroup of $B_{n}$ that acts by signed permutations with an even number of sign changes. The groups $I_{2}(m)$ are the dihedral groups, that is, the symmetry groups of regular $m$-gons. In addition to the infinite families, there are 6 exceptional cases known as $H_{3}, H_{4}, F_{4}, E_{6}, E_{7}$ and $E_{8}$. In each case the subscript of the symbol indicates the rank of the associated reflection arrangement.

Because of the following observation, Conjecture 6 only needs to be proved for irreducible reflection groups.

Lemma 11. If Conjecture 6 is true for the irreducible reflection groups, then it is true for all reflection groups.

Proof. It suffices to consider two reflection groups $\mathcal{W}_{1} \subset \mathrm{GL}\left(\mathbb{R}^{m}\right)$ and $\mathcal{W}_{2} \subset$ $\operatorname{GL}\left(\mathbb{R}^{n-m}\right)$ with fundamental chambers $\mathcal{C}_{1}$ and $\mathcal{C}_{2}$, respectively. For $j=1$, 2, let $b_{j, k}$ be the number of elements $g \in \mathcal{W}_{j}$ for which the projection $\pi_{\mathcal{C}_{j}}\left(g x_{j}\right)$ of a fixed generic point $x_{j} \in \mathcal{C}_{j}$ is $k$-dimensional.

Fix a generic point $x$ in the fundamental chamber $\mathcal{C}=\mathcal{C}_{1} \times \mathcal{C}_{2}$ of the reflection group $\mathcal{W}=\mathcal{W}_{1} \times \mathcal{W}_{2} \subset \mathrm{GL}\left(\mathbb{R}^{n}\right)$. The $k$-dimensional faces of $\mathcal{C}$ are of the form $F_{1} \times F_{2}$, where $F_{1}$ is an $i$-dimensional face of $\mathcal{C}_{1}$ and $F_{2}$ is a $(k-i)$-dimensional face of $\mathcal{C}_{2}$. Therefore, the number of elements $g \in \mathcal{W}$ for which $\pi_{\mathcal{C}}(x)$ is $k$-dimensional is given by the convolution

$$
\sum_{i=0}^{k} b_{1, i} b_{2, k-i}, \quad k=0, \ldots, n .
$$

The reflection arrangement $\mathcal{A}$ associated with $\mathcal{W}$ is the union of two subarrangements $\mathcal{A}_{1}$ and $\mathcal{A}_{2}$ corresponding to $\mathcal{W}_{1}$ and $\mathcal{W}_{2}$, respectively. The normal vectors for the hyperplanes in $\mathcal{A}_{1}$ are in $\mathbb{R}^{m} \times\{0\}^{n-m}$ and those for $\mathcal{A}_{2}$ are in $\{0\}^{m} \times \mathbb{R}^{n-m}$. Our claim now follows because it can be shown that the coefficients of the characteristic polynomial $\chi_{\mathcal{A}}(t)$ also obey the convolution rule in (11) when expressed in terms of the coefficients of the characteristic polynomials $\chi_{\mathcal{A}_{1}}(t)$ and $\chi_{\mathcal{A}_{2}}(t)$; compare [Sta07, Exercise 1.5].

Example 12. Returning to our running example about the non-negative orthant, we see that the characteristic polynomial factors as $t^{2}-2 t+1=(t-1)^{2}$. As mentioned in the above proof, this factorization mirrors the factorization of the non-negative orthant as $[0, \infty) \times[0, \infty)$.

\section{MAin Results}

4.1. Top and bottom coefficients. The case of the top (or leading) coefficient of the characteristic polynomial, corresponding to the fundamental chamber $\mathcal{C}$ itself, 
is well-known. The chambers of the arrangement partition space, and the action of $\mathcal{W}$ on chambers is simply transitive. Therefore, the orbit of any generic point in $\mathbb{R}^{n}$ hits the chamber $\mathcal{C}$ precisely once, and the projection volume for $\mathcal{C}$ is $1 /|\mathcal{W}|$.

The case of the bottom coefficient, the coefficient of the non-zero term of lowest degree, was shown for reflection groups with so-called crystallographic root systems by De Concini and Procesi, and Stembridge [DCP06]. The general case was proven by Denham Den08. The points with lowest-dimensional projection form the polar cone to $\mathcal{C}$, that is, the cone spanned by the normal vectors to the reflecting hyperplanes supporting $\mathcal{C}$ (or in the language of finite root systems, spanned by a set of simple roots). Hence, proving the case of the bottom coefficients amounts to determining the relative spherical volume of the polar cone. We remark that the work of Denham Den08 involves a combinatorial approach that is case-free in that it does not use the classification of the irreducible reflection groups. His approach rests on the fact that the evaluations of the characteristic polynomial of a hyperplane arrangement at \pm 1 have clear combinatorial interpretations.

Since the sum of the coefficients of the characteristic polynomial is equal to the size of the group, we may state the following result.

Proposition 13. Conjecture 6 is true for any reflection arrangement of rank 2, and in particular, for the infinite family $I_{2}(m)$ for which a canonical choice of a fundamental chamber is

$\mathcal{C}\left(I_{2}(m)\right)=\left\{x \in \mathbb{R}^{2}: x_{1} \geq 0, x_{1} \cdot \cos \left(\frac{\pi(m-1)}{m}\right)+x_{2} \cdot \sin \left(\frac{\pi(m-1)}{m}\right) \geq 0\right\}$.

4.2. Weighted projections. In our proof for types $A_{n}, B_{n}$, and $D_{n}$, a connection has to be made between projections in $\mathbb{R}^{n}$ and $\mathbb{R}^{n-1}$. To this end, it will be necessary to work with weighted projections onto the fundamental chamber. For $y \in \mathbb{R}^{n}$, a closed set $\mathcal{K}$ and a weight vector $\omega \in(0, \infty)^{n}$, we define the weighted projection

$$
\pi_{\mathcal{K}}(y ; \omega)=\arg \min _{x \in \mathcal{K}} \sum_{i=1}^{n} \omega_{i}\left(x_{i}-y_{i}\right)^{2},
$$

where $\arg$ min refers to the $x \in \mathcal{K}$ which minimizes the sum.

For example, the weighted projection $\pi_{H}(y ; \omega)$ onto the hyperplane $H=\left\{x_{i}=\right.$ $\left.x_{j}\right\}$ is obtained by replacing the $i$-th and $j$-th components of $y$ with their weighted average

$$
\frac{\omega_{i} y_{i}+\omega_{j} y_{j}}{\omega_{i}+\omega_{j}}
$$

Forming such weighted averages is at the heart of the work in [Mil59], which established Theorem 4 for the symmetric groups $A_{n}$.

Suppose $r_{1}, \ldots, r_{d}$ are the normal vectors of a fundamental chamber $\mathcal{C}$ of a reflection group, that is,

$$
\mathcal{C}=\left\{x \in \mathbb{R}^{n}:\left(r_{i}, x\right) \geq 0 \text { for all } i \in[d]\right\} .
$$

Such a set of normal vectors is also known as a set of simple roots, and they satisfy $\left(r_{i}, r_{j}\right) \leq 0$ for all distinct $i, j \in[d]$; see [GB85, Prop. 4.1.5]. Here, $(x, y)=\sum_{i} x_{i} y_{i}$ denotes the standard inner product of two vectors $x, y \in \mathbb{R}^{n}$. We write $(x, y)_{\omega}=$ $\sum_{i} \omega_{i} x_{i} y_{i}$ for the inner product with respect to a weight vector $\omega$.

The following lemma is concerned with points that are on the "wrong side" of a supporting hyperplane (or a wall) of $\mathcal{C}$. The lemma shows that when a point is on 
the "wrong side", then its (weighted) projection onto $\mathcal{C}$ can be computed by first projecting onto the considered wall.

Lemma 14. Let $\omega \in(0, \infty)^{n}$ be a weight vector and $\phi=\left(1 / \omega_{1}, \ldots, 1 / \omega_{n}\right)$ be the vector of inverted weights. If $\left(r_{i}, r_{j}\right)_{\phi} \leq 0$ for all $j \neq i$, and $y \in \mathbb{R}^{n}$ satisfies $\left(r_{i}, y\right) \leq 0$, then the projection $\pi_{\mathcal{C}}(y ; \omega)$ is in the wall $H=\left\{x \in \mathbb{R}^{n}:\left(r_{i}, x\right)=0\right\}$ of the fundamental chamber and $\pi_{\mathcal{C}}(y ; \omega)=\pi_{\mathcal{C}}\left(\pi_{H}(y ; \omega) ; \omega\right)$.

Proof. A well-known fact about projections on closed convex sets states that $z=$ $\pi_{\mathcal{C}}(y ; \omega)$ if and only if $(y-z, x-z)_{\omega} \leq 0$ for all $x \in \mathcal{C}$.

Consider a point $z \in \mathcal{C} \backslash H$. In particular, $\left(r_{i}, z\right)>0$. Let $\Omega=\operatorname{diag}(\omega) \in \mathbb{R}^{n \times n}$. Pick $\varepsilon>0$ small enough such that $z_{\varepsilon}=z-\varepsilon \Omega^{-1} r_{i}$ satisfies

$$
\left(r_{i}, z_{\varepsilon}\right)=\left(r_{i}, z\right)-\varepsilon \cdot\left(r_{i}, r_{i}\right)_{\phi} \geq 0 .
$$

Then $z_{\varepsilon} \in \mathcal{C}$ because $\left(r_{j}, z_{\varepsilon}\right)=\left(r_{j}, z\right)-\varepsilon\left(r_{j}, r_{i}\right)_{\phi} \geq\left(r_{j}, z\right) \geq 0$ for all $j \neq i$. Moreover, $\left(y-z, z_{\varepsilon}-z\right)_{\omega}=-\varepsilon \cdot\left(r_{i}, y-z\right)>0$ because $\left(r_{i}, y\right)-\left(r_{i}, z\right)<0$. Therefore, $\pi_{\mathcal{C}}(y ; \omega)$ lies in the wall $H$.

Since $\left(r_{i}, \pi_{H}(y ; \omega)\right)=0$, the previous calculation also implies that $\pi_{\mathcal{C}}\left(\pi_{H}(y ; \omega) ; \omega\right)$ is in $H$. Writing $\|x\|_{\omega}^{2}$ for the norm $(x, x)_{\omega}$, we have that for any point $h \in H$,

$$
\|y-h\|_{\omega}^{2}=\left\|y-\pi_{H}(y ; \omega)\right\|_{\omega}^{2}+\left\|\pi_{H}(y ; \omega)-h\right\|_{\omega}^{2} .
$$

Since the projection $\pi_{\mathcal{C}}(y ; \omega)$ is in $H$, it can be determined by minimizing $\|y-h\|_{\omega}^{2}$ for $h \in \mathcal{C} \cap H$. By (3), we may instead minimize $\left\|\pi_{H}(y ; \omega)-h\right\|_{\omega}^{2}$. This latter minimization, however, also yields $\pi_{\mathcal{C}}\left(\pi_{H}(y ; \omega) ; \omega\right)$ because $\pi_{\mathcal{C}}\left(\pi_{H}(y ; \omega) ; \omega\right) \in H$. Hence, $\pi_{\mathcal{C}}(y ; \omega)=\pi_{\mathcal{C}}\left(\pi_{H}(y ; \omega) ; \omega\right)$, as claimed.

Example 15 (Groups of Type $A_{n}$ ). The group $A_{n-1}$ has simple $\operatorname{roots} r_{i}=e_{i+1}-e_{i}$, $i=1, \ldots, n-1$. They define the chamber

$$
\mathcal{C}\left(A_{n-1}\right)=\left\{x \in \mathbb{R}^{n}: x_{1} \leq \cdots \leq x_{n}\right\}
$$

from Theorem 3. Each root has only two non-zero entries, and $\left(r_{i}, r_{i+1}\right)_{\phi}=$ $-1 / \omega_{i+1}<0$ and $\left(r_{i}, r_{j}\right)_{\phi}=0$ if $j \geq i+2$. Hence, the condition of Lemma 14 that requires $\left(r_{i}, r_{j}\right)_{\phi} \leq 0$ for all $i \neq j$ holds for any weight vector $\omega$.

Example 16 (Groups of type $B_{n}$ ). The group $B_{n}$ has simple roots $r_{1}=e_{1}$ and $r_{i}=e_{i}-e_{i-1}, i=2, \ldots, n$. They define the chamber

$$
\mathcal{C}\left(B_{n}\right)=\left\{x \in \mathbb{R}^{n}: 0 \leq x_{1} \leq \cdots \leq x_{n}\right\} .
$$

Again the condition of Lemma 14 holds for any weight vector $\omega$ because $\left(r_{1}, r_{2}\right)_{\phi}=$ $-1 / \omega_{1}<0,\left(r_{i}, r_{i+1}\right)_{\phi}=-1 / \omega_{i}<0$ and $\left(r_{i}, r_{j}\right)_{\phi}=0$ if $j \geq i+2$.

Example 17 (Groups of type $D_{n}$ ). A natural choice of simple roots for the group $D_{n}$ is $r_{1}=e_{1}+e_{2}, r_{2}=e_{2}-e_{1}$ and $r_{i}=e_{i}-e_{i-1}, i=3, \ldots, n$. They define the chamber

$$
\mathcal{C}\left(D_{n}\right)=\left\{x \in \mathbb{R}^{n}:\left|x_{1}\right| \leq x_{2} \cdots \leq x_{n}\right\} .
$$

In this case, the requirement that $\left(r_{i}, r_{j}\right)_{\phi} \leq 0$ for all $i \neq j$ appearing in Lemma 14 does present a condition on a positive weight vector $\omega$. Namely, it needs to hold that $\omega_{1} \geq \omega_{2}$ because $\left(r_{1}, r_{2}\right)_{\phi}=1 / \omega_{2}-1 / \omega_{1}$. 


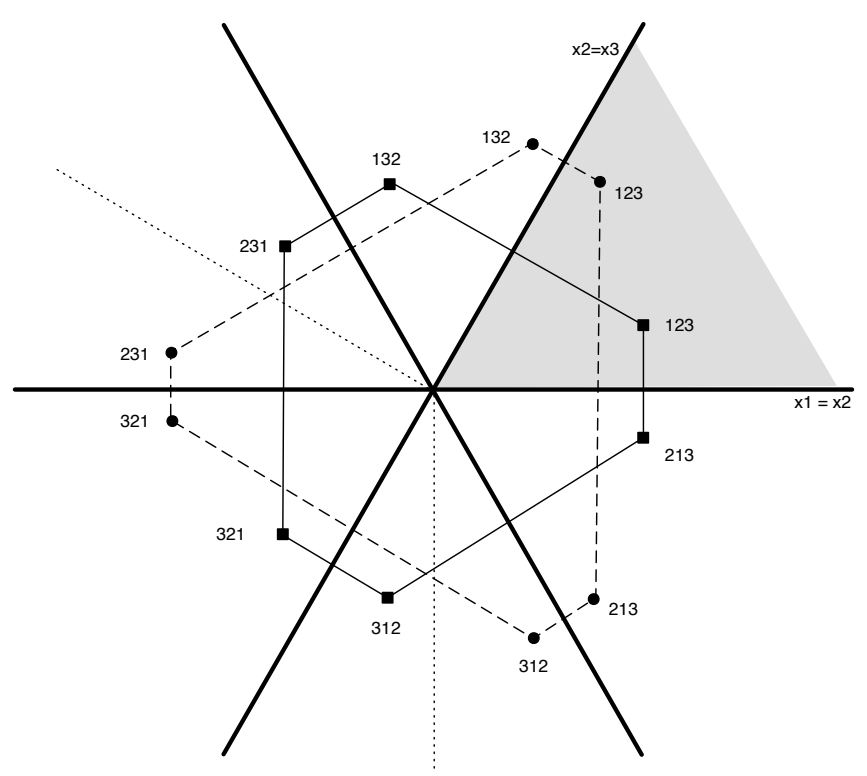

Figure 1. Orbits of two points under the reflections of $A_{2}$. The projection chambers are defined by the chosen fundamental chamber (shaded) and the dotted half lines. Moving counter-clockwise, the round point is mapped only once into the first 1-dimensional projection chamber, whereas the square point lands in it twice.

4.3. Groups of type $A_{n}, B_{n}, D_{n}$. In this section, we prove Theorem 4 that is, we show that Conjecture 6 holds for the three infinite families of real irreducible reflection groups, $A_{n}, B_{n}$, and $D_{n}$. The result is known to hold for the groups $A_{n}$; recall Theorem 3. This case was first proven in Mil59. Our work provides and generalizes a geometric version of this proof.

One difficulty in proving Theorem 4 is illustrated in Figure 1, which concerns the group $A_{2}$. The figure shows the orbits of two choices of a generic point $x$. We see that varying $x$ can change the dimensionality of the projection of a particular point $g x$ in the orbit. Nevertheless, the number of points in the orbit that have a projection of fixed dimension is independent of the choice of $x$.

Our proof relies on the well-known factorization of the characteristic polynomial of a reflection arrangement:

$$
\chi_{\mathcal{A}}(t)=\left(t-e_{1}\right)\left(t-e_{2}\right) \cdots\left(t-e_{n}\right),
$$

where the $e_{i}$ s are known as the exponents of the group; see [OT92, Thm. 4.137]. For the considered groups, we will be able to build an induction argument around this factorization by decomposing each group into $e_{n}$ pieces, where each are in oneto-one correspondence with a reflection group of lower rank. Here, $e_{n}$ is the highest exponent of the group. 
Theorem 18. Let $\mathcal{W}$ be a reflection group of type $A_{n}$ or $B_{n}$, and let $\omega \in(0, \infty)^{n}$ be any weight vector. For a generic point $x$ in the fundamental chamber $\mathcal{C}$ of $\mathcal{W}$, the number of group elements $g \in \mathcal{W}$ with $\pi_{\mathcal{C}}(g x ; g \omega) k$-dimensional equals the absolute value of the coefficient of $t^{k}$ in the characteristic polynomial $\chi_{\mathcal{W}}(t)$.

Proof. We argue by induction on $n$, the index of the group. The claim is easily seen to be true for the induction base $n=1$. Although the method of proof for the induction step is the same, we must break up the two families separately.

Case A. The irreducible reflection group $A_{n-1}$ is isomorphic to the group of permutations of $n$ characters and has the canonical fundamental chamber $\mathcal{C}\left(A_{n-1}\right)$ discussed in Example 15. We abbreviate the projection map onto this chamber by $\pi_{n-1}$. The reflection arrangement corresponding to $A_{n-1}$ is the collection of hyperplanes

$$
\mathcal{A}_{n-1}=\left(\left\{x_{i}-x_{j}=0\right\}: 1 \leq i<j \leq n\right),
$$

also known as the braid arrangement. It has characteristic polynomial

$$
\chi_{n-1}^{A}(t)=t(t-1) \cdots(t-n+1) .
$$

Fix a generic point $x \in \mathcal{C}\left(A_{n-1}\right)$. In particular, the coordinates of $x$ are ordered as $x_{1}<\cdots<x_{n}$. Let

$$
A_{n-1, n}=\left\{g \in A_{n-1}:(g x)_{n}=x_{n}\right\},
$$

and, for $j=1, \ldots, n-1$,

$$
A_{n-1, j}=\left\{g \in A_{n-1}:(g x)_{i}=x_{n} \text { and }(g x)_{i+1}=x_{j} \text { for some } i \in[n]\right\} .
$$

Clearly, the sets $A_{n-1,1}, \ldots, A_{n-1, n}$ form a partition of $A_{n-1}$. In what follows, $\rho_{j}: \mathbb{R}^{n} \rightarrow \mathbb{R}^{n-1}$ denotes the restriction map omitting the $j$-th coordinate.

The set $A_{n-1, n}$ is a subgroup isomorphic to $A_{n-2}$. For $g \in A_{n-1, n}$, define $\bar{g}$ to be the element of $A_{n-2}$ for which $\rho_{n}(g x)=\bar{g} \rho_{n}(x)$. Then $\pi_{n-1}(g x, g \omega)$ is $k$ dimensional if and only if $\pi_{n-2}\left(\bar{g} \rho_{n}(x), \bar{g} \rho_{n}(\omega)\right)$ is $(k-1)$-dimensional with respect to the chamber $\mathcal{C}\left(A_{n-2}\right)$. By the induction hypothesis, the number of $k$-dimensional elements in $A_{n-1, n}$ equals the coefficient of $t^{k}$ in $t \cdot \chi_{n-2}^{A}(t)$ in absolute value.

If $j<n$, then $A_{n-1, j}$ is again in 1:1-correspondence to $A_{n-2}$. Let $g \in A_{n-1, j}$ satisfy $(g x)_{i}=x_{n}$ and $(g x)_{i+1}=x_{j}$. The corresponding element $\bar{g} \in A_{n-2}$ is determined by $\rho_{i}(g x)=\bar{g} \rho_{n}(x)$. Let $H=\left\{x_{i}=x_{i+1}\right\}$. Then the $i$-th and the $(i+1)$-st components in $\pi_{H}(g x ; g \omega)$ are equal; recall (2). Since $(g x)_{i}=x_{n}>x_{j}=$ $(g x)_{i+1}$ the point $g x$ is on the "wrong side" of the wall $H$. Lemma 14 implies that $\pi_{n-1}(g x, g \omega)$ is $k$-dimensional if and only if $\pi_{n-1}\left(\pi_{H}(g x ; g \omega), g \omega\right)$ is $k$-dimensional. Since $\pi_{H}(g x ; g \omega)=g \pi_{K}(x ; \omega)$ for $K=\left\{x_{j}=x_{n}\right\}$, this in turn occurs if and only if $\pi_{n-2}\left(\bar{g} \rho_{n}\left(\pi_{K}(x ; \omega)\right), \bar{g} \bar{\omega}\right)$ is $k$-dimensional. The new weights $\bar{\omega} \in(0, \infty)^{n-1}$ satisfy $\bar{\omega}_{l}=\omega_{l}$ for $l<j, \bar{\omega}_{j}=\omega_{j}+\omega_{n}$ and $\bar{\omega}_{l}=\omega_{l+1}$ for $j<l<n$. By the induction hypothesis, the number of $k$-dimensional elements in $A_{n-1, j}$ equals the absolute value of the coefficient of $t^{k}$ in $\chi_{n-2}^{A}(t)$.

Adding up the count of $k$-dimensional elements in each one of the sets $A_{n-1, j}$, we obtain the number of $k$-dimensional elements in $A_{n-1}$. This number is thus equal in absolute value to the coefficient of $t^{k}$ in

$$
t \cdot \chi_{n-2}^{A}(t)-(n-1) \cdot \chi_{n-2}^{A}(t)=\chi_{n-1}^{A}(t) .
$$


Case B. The irreducible reflection group $B_{n}$ acts by signed permutations and has the canonical fundamental chamber $\mathcal{C}\left(B_{n}\right)$ discussed in Example 16. We denote the projection map onto this chamber by $\pi_{n}$. The reflection arrangement corresponding to $B_{n}$ consists of the hyperplanes

$$
\mathcal{B}_{n}=\left(\left\{x_{i}-x_{j}=0\right\},\left\{x_{i}+x_{j}=0\right\},\left\{x_{k}=0\right\}: 1 \leq i<j \leq n, 1 \leq k \leq n\right) .
$$

The characteristic polynomial for the $B_{n}$ arrangement is

$$
\chi_{n}^{B}(t)=(t-1)(t-3) \cdots(t-(2 n-1)) .
$$

Fix a generic point $x \in \mathcal{C}\left(B_{n}\right)$, which has coordinates $0<x_{1}<\cdots<x_{n}$. For a partition of $B_{n}$, let

$$
\begin{aligned}
& B_{n, n}=\left\{g \in B_{n}:(g x)_{n}=x_{n}\right\} \text { and } \\
& B_{n, 0}=\left\{g \in B_{n}:(g x)_{1}=-x_{n}\right\},
\end{aligned}
$$

and, for $j=1, \ldots n-1$,

$$
B_{n, j}=\left\{g \in B_{n}: \begin{array}{l}
(g x)_{i}=x_{n} \text { and }(g x)_{i+1}=x_{j}, \text { or } \\
(g x)_{i+1}=-x_{n} \text { and }(g x)_{i}=-x_{j}
\end{array} \quad \text { for some } i \in[n-1]\right\}
$$

and

$$
B_{n,-j}=\left\{g \in B_{n}: \begin{array}{l}
(g x)_{i}=x_{n} \text { and }(g x)_{i+1}=-x_{j}, \text { or } \\
(g x)_{i+1}=-x_{n} \text { and }(g x)_{i}=x_{j}
\end{array} \quad \text { for some } i \in[n-1]\right\} .
$$

The sets $B_{n, 0}, B_{n, n}, B_{n, j}, B_{n,-j}, 1 \leq j \leq n-1$, indeed form a partition of $B_{n}$.

The set $B_{n, n}$ is a subgroup isomorphic to $B_{n-1}$. For $g \in B_{n, n}$, define $\bar{g}$ to be the element of $B_{n-1}$ for which $\rho_{n}(g x)=\bar{g} \rho_{n}(x)$. Then $\pi_{n}(g x, g \omega)$ is $k$-dimensional if and only if $\pi_{n-1}\left(\bar{g} \rho_{n}(x), \bar{g} \rho_{n}(\omega)\right)$ is $(k-1)$-dimensional with respect to the chamber $\mathcal{C}\left(B_{n-1}\right)$. By the induction hypothesis, the number of $k$-dimensional elements in $B_{n, n}$ equals the coefficient of $t^{k}$ in $t \cdot \chi_{n-1}^{B}(t)$ in absolute value.

The set $B_{n, 0}$ is in 1:1-correspondence with $B_{n-1}$. Let $H=\left\{x_{n}=0\right\}$. Then $\pi_{H}(x ; \omega)$ is a vector with its $n$-th coordinate equal to 0 . Since $(g x)_{1}=-x_{n}$ for $g \in B_{n, 0}$, the first coordinate of $g \pi_{H}(x ; \omega)$ is 0 . This follows because $x_{n}$ is the largest entry in $x$. Define $\bar{g}$ to be the element of $B_{n-1}$ such that $\rho_{1}(g x)=\bar{g} \rho_{n}(x)$. Lemma 14 implies that $\pi_{n}(g x, g \omega)$ is $k$-dimensional if and only if $\pi_{n}\left(g \pi_{H}(x ; \omega), g \omega\right)$ is $k$-dimensional. This in turn occurs if and only if $\pi_{n-1}\left(\bar{g} \rho_{n}\left(\pi_{H}(x ; \omega)\right), \bar{g} \bar{\omega}\right)$ is $k$-dimensional, where the new weights $\bar{\omega} \in(0, \infty)^{n-1}$ satisfy $\bar{\omega}_{l}=\omega_{l+1}$ for $l<n$.

For $1 \leq j \leq n-1$, the sets $B_{n, j}$ and $B_{n,-j}$ are again in 1:1-correspondence with $B_{n-1}$. If $g$ is an element in $B_{n, j}$ or $B_{n,-j}$, then $g$ satisfies one of the four defining relationships above. In each case, the point $x$ is mapped by $g$ to the "wrong side" of the bounding wall $\left\{x_{i}=x_{i+1}\right\}$, namely, $(g x)_{i}>(g x)_{i+1}$. Note also that $(g x)_{i}$ and $(g x)_{i+1}$ appear with equal signs in $B_{n, j}$ and opposite signs in $B_{n,-j}$. Therefore, we may apply Lemma 14 and average coordinates and add weights as in the $A$-case. It follows that the number of $k$-dimensional elements in either $B_{n, j}$ or $B_{n,-j}$ equals the absolute value of the coefficient of $t^{k}$ in $\chi_{n-1}^{B}(t)$. As there are a total of $2 n-2$ sets of the form $B_{n, j}$ or $B_{n,-j}$, along with the contributions from $B_{n, n}$ and $B_{n, 0}$, we have shown that the total number of $k$-dimensional elements in $B_{n}$ is the absolute value of the coefficient of $t^{k}$ in

$$
t \cdot \chi_{n-1}^{B}(t)-(2 n-1) \cdot \chi_{n-1}^{B}(t)=\chi_{n}^{B}(t),
$$

which was our claim. 
Our second main result concerns the groups of type $D_{n}$. As is clear from Example 17, the use of arbitrary weighted projections is no longer possible. Nevertheless, we can prove Conjecture 6] in the original unweighted version that is of most interest. Compared to the $A$ - and the $B$-cases, a notable difference in the proof below is that we do not break up $D_{n}$ into copies of $D_{n-1}$.

Theorem 19. For a generic point $x$ in a fundamental chamber $\mathcal{C}$ of the reflection group $D_{n}$, the number of elements $g \in D_{n}$ that have $\pi_{\mathcal{C}}(g x) k$-dimensional equals the absolute value of the coefficient of $t^{k}$ in the characteristic polynomial $\chi_{n}^{D}(t)$.

Proof. The irreducible reflection group $D_{n}$ acts by signed permutations with an even number of sign changes. It has the canonical fundamental chamber $\mathcal{C}\left(D_{n}\right)$ discussed in Example 17. We denote the projection onto this chamber by $\pi_{n}^{D}$. The reflection arrangement corresponding to $D_{n}$ is the collection of hyperplanes

$$
\mathcal{D}_{n}=\left(\left\{x_{i}-x_{j}=0\right\},\left\{x_{i}+x_{j}=0\right\}: 1 \leq i<j \leq n\right) .
$$

The characteristic polynomial of this arrangement is

$$
\chi_{n}^{D}(t)=(t-1)(t-3) \cdots(t-2 n+3)(t-n+1) .
$$

We now adopt the same partitioning method as in the proof of Theorem 18. The partition of $D_{n}$ results from a coarsening of the decomposition in the $B$-case. Fix a generic point $x \in \mathcal{C}\left(D_{n}\right)$. In particular, $0<\left|x_{1}\right|<x_{2}<\cdots<x_{n}$. Let

$$
D_{n, n}=\left\{g \in D_{n}:(g x)_{n}=x_{n} \text { or }(g x)_{1}=-x_{n}\right\} \text {. }
$$

For $j=1, \ldots n-1$, let

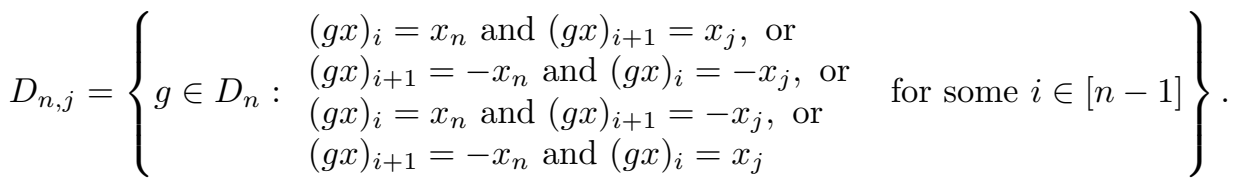

The sets $D_{n, j}, 1 \leq j \leq n$, partition $D_{n}$.

The set $D_{n, n}$ is a subgroup isomorphic to $B_{n-1}$. The explicit isomorphism is given by mapping $g \in D_{n, n}$ to $\bar{g} \in B_{n-1}$ defined as follows. If $g \in D_{n, n}$ satisfies $(g x)_{n}=x_{n}$, then we define $\bar{g} \in B_{n-1}$ by requiring that $\rho_{n}(g x)=\bar{g} \rho_{n}(x)$. Note that $\bar{g}$ is a signed permutation with an even number of sign changes. If $g \in D_{n, n}$ satisfies $(g x)_{1}=-x_{n}$, then we define $\bar{g} \in B_{n-1}$ by $\rho_{1}(g x)=\bar{g} \rho_{n}(x)$. Now $\bar{g}$ is a signed permutation with an odd number of sign changes. With this correspondence between $D_{n, n}$ and $B_{n-1}$, it holds that $\pi_{n}^{D}(g x)$ is $k$-dimensional if and only if $\pi_{n-1}^{B}\left(\bar{g} \rho_{n}(x)\right)$ is $(k-1)$-dimensional. Here, $\pi_{n-1}^{B}$ stands for the projection onto the fundamental chamber $\mathcal{C}\left(B_{n-1}\right)$. By Theorem 18, the absolute value of the coefficient of $t^{k}$ in $t \cdot \chi_{n-1}^{B}(t)$ enumerates the $k$-dimensional elements in $D_{n, n}$.

For $1 \leq j \leq n-1$, the sets $D_{n, j}$ are again in 1:1-correspondence with $B_{n-1}$. An element $g \in D_{n, j}$ satisfies one of the four defining relationships above. In each case, the point $x$ is mapped by $g$ to the "wrong side" of the bounding wall $\left\{x_{i}=x_{i+1}\right\}$, namely, $(g x)_{i}>(g x)_{i+1}$. Note also that $(g x)_{i}$ and $(g x)_{i+1}$ may appear with both equal and opposite signs. Being in an unweighted situation, or rather in a situation with weight vector $\omega=(1, \ldots, 1)$, we may apply Lemma 14 and average coordinates and add weights as in the $A$ - and the $B$-cases. By Theorem 18 , we obtain that the number of $k$-dimensional elements in $D_{n, j}$ equals the absolute value of the coefficient of $t^{k}$ in $\chi_{n-1}^{B}(t)$. There being a total of $n-1$ sets of the form $D_{n, j}$, along with the 
contribution from $D_{n, n}$, the total number of $k$-dimensional elements in $D_{n}$ is seen to be the absolute value of the coefficient of $t^{k}$ in

$$
t \cdot \chi_{n-1}^{B}(t)-(n-1) \cdot \chi_{n-1}^{B}(t)=\chi_{n}^{D}(t),
$$

as claimed.

Remark 20. Our method of proof breaks a Coxeter group into copies of a suitable subgroup. One might hope to extend this to a general method for all reflection groups. However, this is not possible, as can be seen for example from the fact that removing the highest factor $\left(t-e_{n}\right)$ in the factorization in (4) need not yield the characteristic polynomial of a reflection group OT92, Section 6.5]. In this context, see also [BI98, where it is shown that if $\mathcal{W}^{\prime}$ is a parabolic subgroup of a reflection group $\mathcal{W}$, then the characteristic polynomial $\chi_{\mathcal{W}^{\prime}}(t)$ divides $\chi_{W}(t)$ if and only if $\mathcal{W}$ is of type $A_{n}$ or $B_{n}$, or $\mathcal{W}^{\prime}$ is of rank 1.

\section{EXCEPTIONAL GROUPS}

The proof method used in the previous section decomposes a reflection group into copies of a reflection subgroup and proceeds inductively. The method, however, breaks down for the exceptional irreducible reflection groups; recall Remark 20. Nevertheless, we believe that Conjecture 6 also holds for these groups. In the remainder of this section we describe simulation evidence that supports this belief.

We tested our conjecture for the groups $H_{3}, H_{4}, F_{4}, E_{6}$, and $E_{7}$. The only other exceptional irreducible group, $E_{8}$, was too large for our implementation. For each considered group, we randomly chose points in the fundamental chamber and counted the number of points with $k$-dimensional projections in the resulting orbits. We considered 1000 randomly chosen points for the groups of rank at most 6 and 50 points for $E_{7}$. Conjecture 6 held in all tested cases; that is, in each test orbit the number of group elements with $k$-dimensional projection was equal to the absolute values of the coefficient of $t^{k}$ in the characteristic polynomial $\chi(t)$.

Suppose that the considered fundamental chamber

$$
\mathcal{C}=\left\{x \in \mathbb{R}^{n}:\left(r_{i}, x\right) \geq 0 \text { for all } i \in[d]\right\}
$$

is defined by the simple roots $r_{1}, \ldots, r_{d}$. Let $V$ be the linear space spanned by $\left\{r_{1}, \ldots, r_{d}\right\}$. Define the dual roots $s_{1}, \ldots, s_{d} \in V$ by requiring that $\left(s_{i}, r_{j}\right)=\delta_{i j}$. Then $\mathcal{C} \cap V$ is the simplicial cone spanned by $\left\{s_{1}, \ldots, s_{d}\right\}$; see [GB85, §4.2]. Our procedure for generating random points in the fundamental chamber is based on this spanning set representation; namely, it picks points uniformly at random from the convex hull of $\left\{s_{1}, \ldots, s_{d}\right\}$.

Given a point $x \in \mathcal{C}$, we need to visit each point in the orbit of $x$. We accomplished this using the traverse function from John Stembridge's Maple package coxeter [Ste. In order to calculate the dimensions of the projections $\pi_{\mathcal{C}}(g x)$ quickly, we precomputed all projection chambers; that is, for each face $F$ we computed an inequality representation of the polyhedral cone of all points projected on $F$. This precomputation is based on the following lemma, which simply states that the points which project to a given face $F$ form the polyhedral cone spanned by the face itself and the opposites to the defining normals of the face. The conversion to an inequality representation was done with the software polymake GJ00. 
Lemma 21. Let $K \subseteq[d]$ be of cardinality $k=|K|$. Let

$$
F=\left\{x \in \mathbb{R}^{n}:\left(r_{i}, x\right)=0 \text { for all } i \in K,\left(r_{i}, x\right) \geq 0 \text { for all } i \in[d] \backslash K\right\}
$$

be the $k$-dimensional face of the fundamental chamber associated with $K$. Then the set of all points $x \in V$ with projection $\pi_{\mathcal{C}}(x) \in F$ is the polyhedral cone spanned by $\left\{-r_{i}: i \in K\right\} \cup\left\{s_{i}: i \in[d] \backslash K\right\}$.

While our simulations offer strong evidence for the validity of Conjecture 6 , consideration of a single (or several) random point(s) unfortunately does not prove the conjecture. The difficulty lies in the fact that the orbits of different points in the fundamental chamber behave differently with respect to projection chambers. We refer to Figure 1, where the orbits of two distinct points from the fundamental chamber of $A_{2}$ are shown.

Remark 22. The fact that the orbits behave differently with respect to projection chambers also makes it difficult to connect our interpretation of the coefficients with others that have appeared in the literature. For example, $\left|a_{k}\right|$ is also known to be the number of group elements in $\mathcal{W}$ that leave fixed all points of some linear space of dimension $n-k$; see [ST54, Section 5]. These two interpretations do not coincide, and it would thus be interesting to establish a bijection between the different partitions of $\mathcal{W}$. The partition of $\mathcal{W}$ obtained through Conjecture 6 will however depend on the orbit type of $x$.

A computational approach towards a full proof of Conjecture [6 could proceed by decomposing the fundamental chamber $\mathcal{C}$ of each of the six exceptional irreducible reflection groups by orbit type. For a given group $\mathcal{W}$, define two points $x$ and $y$ equivalent if for all group elements $g \in \mathcal{W}$, the projection $\pi_{\mathcal{C}}(g x)$ is in the relative interior of the same face of $\mathcal{C}$ as $\pi_{\mathcal{C}}(g y)$. By a decomposition by orbit type we mean a polyhedral subdivision of $\mathcal{C}$ that corresponds to equivalence classes of the relation just defined. A computer proof for $\mathcal{W}$ would then be complete upon consideration of the orbit of a single point from each cone in the subdivision.

While such a computer proof may be feasible in a language faster than Maple, it would be more desirable if a case-free proof of Conjecture 6 could be found, eliminating in particular a separate treatment of the exceptional groups.

\section{ACKNOWLEDGMENT}

We are grateful to Bernd Sturmfels for introducing the authors.

\section{REFERENCES}

[BB05] Anders Björner and Francesco Brenti, Combinatorics of Coxeter groups, Graduate Texts in Mathematics, vol. 231, Springer, New York, 2005. MR.2133266 (2006d:05001)

[BGW03] Alexandre V. Borovik, I. M. Gelfand, and Neil White, Coxeter matroids, Progress in Mathematics, vol. 216, Birkhäuser Boston Inc., Boston, MA, 2003. MR.1989953 (2004i:05028)

[BI98] H. Barcelo and E. Ihrig, Modular elements in the lattice $L(A)$ when $A$ is a real reflection arrangement, Discrete Math. 193 (1998), no. 1-3, 61-68. Selected papers in honor of Adriano Garsia (Taormina, 1994). MR1661362 (2000e:05166)

[DCP06] Corrado De Concini and Claudio Procesi, A curious identity and the volume of the root spherical simplex, Atti Accad. Naz. Lincei Cl. Sci. Fis. Mat. Natur. Rend. Lincei (9) Mat. Appl. 17 (2006), no. 2, 155-165. With an appendix by John R. Stembridge. MR2238373 (2007e:20079) 
[Den08] Graham Denham, A note on De Concini and Procesi's curious identity, Atti Accad. Naz. Lincei Cl. Sci. Fis. Mat. Natur. Rend. Lincei (9) Mat. Appl. 19 (2008), no. 1, 59-63. MR2383561(2009a:20064)

[FR07] Sergey Fomin and Nathan Reading, Root systems and generalized associahedra, Geometric combinatorics, IAS/Park City Math. Ser., vol. 13, Amer. Math. Soc., Providence, RI, 2007, pp. 63-131. MR2383126

[GB85] L. C. Grove and C. T. Benson, Finite reflection groups, second ed., Graduate Texts in Mathematics, vol. 99, Springer-Verlag, New York, 1985. MR777684 (85m:20001)

[GJ00] Ewgenij Gawrilow and Michael Joswig, Polymake: a framework for analyzing convex polytopes, Polytopes - combinatorics and computation (Oberwolfach, 1997), DMV Sem., vol. 29, Birkhäuser, Basel, 2000, pp. 43-73. MR.1785292 (2001f:52033)

[Hum90] James E. Humphreys, Reflection groups and Coxeter groups, Cambridge Studies in Advanced Mathematics, vol. 29, Cambridge University Press, Cambridge, 1990. MR:1066460 (92h:20002)

[Kan01] Richard Kane, Reflection groups and invariant theory, CMS Books in Mathematics/Ouvrages de Mathématiques de la SMC, 5, Springer-Verlag, New York, 2001. MR.1838580 (2002c:20061)

[Mil59] R. E. Miles, The complete amalgamation into blocks, by weighted means, of a finite set of real numbers, Biometrika 46 (1959), 317-327. MR0112167(22:3022)

[OT92] Peter Orlik and Hiroaki Terao, Arrangements of hyperplanes, Grundlehren der Mathematischen Wissenschaften [Fundamental Principles of Mathematical Sciences], vol. 300, Springer-Verlag, Berlin, 1992. MR.1217488(94e:52014)

[RWD88] Tim Robertson, F. T. Wright, and R. L. Dykstra, Order restricted statistical inference, Wiley Series in Probability and Mathematical Statistics: Probability and Mathematical Statistics, John Wiley \& Sons Ltd., Chichester, 1988. MR961262(90b:62001)

[SS05] Mervyn J. Silvapulle and Pranab K. Sen, Constrained statistical inference, Inequality, order, and shape restrictions, Wiley Series in Probability and Statistics, WileyInterscience [John Wiley \& Sons], Hoboken, NJ, 2005. MR2099529(2005k:62004)

[ST54] G. C. Shephard and J. A. Todd, Finite unitary reflection groups, Canadian J. Math. 6 (1954), 274-304. MR0059914 (15,600b)

[Sta07] R. P. Stanley, An introduction to hyperplane arrangements, Geometric combinatorics, IAS/Park City Math. Ser., vol. 13, Amer. Math. Soc., Providence, RI, 2007, pp. 389496. MR 2383131

[Ste] John Stembridge, The Coxeter/Weyl package for the computer algebra system Maple, available online at http://www.math.lsa.umich.edu/ ${ }^{j}$ jrs/maple.html.

Department of Statistics, University of Chicago, 5734 S. University Avenue, Chicago, ILLINOIS 60637-1514

Departments of Mathematics and Computer Science, University of Chicago, 5734

S. University Avenue, Chicago, Illinois 60637-1538 